EPJ Web of Conferences 66, 01018 (2014)

DOI: $10.1051 /$ epjconf/ 20146601018

(C) Owned by the authors, published by EDP Sciences, 2014

\title{
Probing the nuclear equation-of-state and the symmetry energy with heavy-ion collisions
}

\author{
Giuseppe Verde ${ }^{1, a}$ \\ ${ }^{1}$ Istituto Nazionale di Fisica Nucleare, Sezione di Catania, 64 Via Santa Sofia, I-95123, Catania, Italy
}

\begin{abstract}
The present status of studies aimed at constraining the nuclear equation of state with heavy-ion collision dynamics is presented. Multifragmentation phenomena, including their isotopic distributions, charge correlations and emission time-scales, may revel the existence of liquid-gas transitions in the phase diagram. Exploring the isotopic degree of freedom in nuclear dynamics is then required in order to constrain the equation of state of asymmetric nuclear matter which presently represents a major priority due to its relevance to both nuclear physics and astrophysics. Some observables that have successfully constrained the density dependence of the symmetry energy are presented, such as neutron-proton yield ratios and isospin diffusion and drift phenomena. The reported results and status of the art is discussed by also considering some of the present problems and some future perspectives for the heavy-ion collision community.
\end{abstract}

\section{Introduction}

Accessing the properties of nuclear matter, constituted by an infinite number of neutrons and protons interacting via Van der Waals-like forces, is one of the main research topics in nuclear physics. Theoretical predictions indicate that at moderate temperatures and densities, below the saturation density, $\rho_{0} \approx 0.17 \mathrm{fm}^{-3}$, there may be a liquid-gas phase transition above which nucleons are not bound anymore [1-3]. At higher temperature hadronic matter is expected to undergo a transition to a quarkgluon plasma [4] with is presently under study at CERN and RHIC facilities. Experimentally it is a matter of debate whether signals of phase transitions have been actually observed, due to the difficulties in extrapolating bulk matter properties from measured observables in heavy-ion collisions. This contribution will focus on quest for the Equation of State (EoS) of nuclear matter as it can be accessed with heavy-ion collisions (HIC) at intermediate energies $\left(\mathrm{E}_{\text {beam }} / \mathrm{A}=20-100 \mathrm{MeV}\right)$. How much energy is needed to compress hadronic matter? Answering this questions is also important for the understanding of astrophysical observations on supernovae explosions and neutron star properties. The nuclear EoS, $E(\rho, T, \delta)$, is the dependence of the compressional energy density $E$, density $\rho=\rho_{n}+\rho_{p}$, temperature $T$, and isospin asymmetry $\delta=\left(\rho_{n}-\rho_{p}\right) /\left(\rho_{n}+\rho_{p}\right)$. This article shows that HIC are unique tools to study the EoS. The extracted results need to be compared to astrophysical observation studies and this represents an priority for the scientific community.

This is an Open Access article distributed under the terms of the Creative Commons Attribution License 2.0, which permits unrestricted use, distribution, and reproduction in any medium, provided the original work is properly cited. 

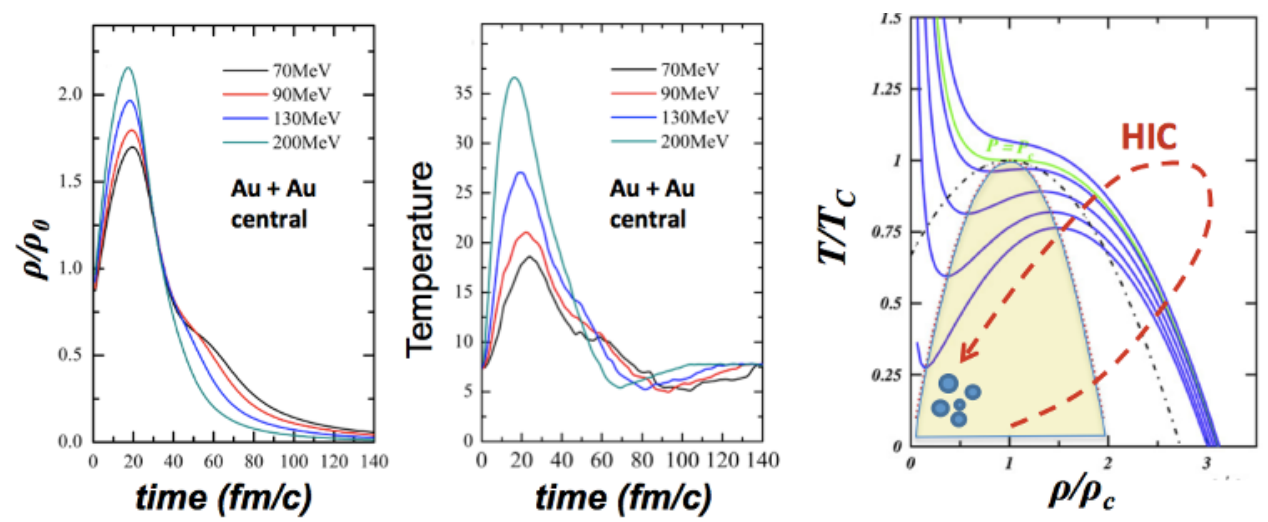

Figure 1. Left and central panels: time evolution of density $\rho$ (normalized to saturation density $\rho_{0}$ ) and temperature predicted in QMD simulations of central $\mathrm{Au}+\mathrm{Au}$ collisions at different incident energies per nucleon. Right panel: isobars of the nuclear EoS for Skyrme interactions [3] (see text for description).

\section{The Equation of State in laboratory nuclear reactions}

Heavy-ion collisions allow ones to study the nuclear equation of state (EoS) under laboratory controlled conditions. The left and middle panels of Fig. 1 show the time evolution of density $\rho$ (normalized to saturation density $\rho_{0}=0.17 \mathrm{fm}^{-3}$ ) and temperature $T$, respectively, of the nuclear systems produced in central $\mathrm{Au}+\mathrm{Au}$ collisions at different incident energies per nucleon. The curves are obtained with Quantum Molecular Dynamics model simulations of Ref. [5]. By increasing the beam energy one can produce nuclear systems at higher densities (supra-saturation densities, $\rho / \rho_{0}>1$ ) in the early stage of the reaction (around $t \approx 20 \mathrm{fm} / \mathrm{c}$ ). The system later expands to sub-saturation densities $\left(\rho / \rho_{0}<1\right)$. One can thus explore different regions of the EoS diagram, schematically shown in the case of Skyrme interactions on the right panel of Fig. 1. The different lines show temperatures, $T / T_{c}$ (where $T_{c}=16 \mathrm{MeV}$ is the critical temperature), as a function of densities, $\rho / \rho_{c}$, for constant values of pressure [1-3]. The red thick dashed line shows a possible path followed by the nuclear system during the reaction. If the system lives long-enough in the so-called spinodal instability region (delimited by the shaded area in the figure, where the derivative of pressure with respect to density is negative) instabilities may develop and multifragmentation takes place: the systems breakup into intermediate mass fragments (IMF) with $\mathrm{Z}>2$ [6]. Multifragmentation has long been sought as an experimental signature of liquid-gas phase coexistence in nuclear matter [7]. Heavy fusion and fission residues are commonly associated to samples of the "liquid phase", while systems composed of isolated nucleons and light clusters, typically produced in most violent collisions, are commonly associated to sample of a nuclear "gas" [7]. HIC collisions studies need to be performed with $4 \pi$ detectors [8] allowing one to completely reconstruct the collision event and detect all produced fragments.

Over the past twenty years several signals of phase transitions have been studied (see [3] for a recent review): caloric curves[9], critical phenomena [10]; bimodality [11]; scalings in charge partitions [12]. These successful studies have faced even conceptual and experimental difficulties linked to the difficulty in using equilibrium concepts (such as temperature, heat capacity and phase transitions) in a quickly evolving finite system where mean-field and nucleon-nucleon collision dynamics play a key role. These difficulting make it challenging to answer the famous question: Is multifragmentation

\footnotetext{
a e-mail: giuseppe.verde@ct.infn.it
} 
a condensation phenomenon or rather a shattering of glass? [13]. The most recent attempt in that direction is presented in a work by the Indra collaboration [14] where central Xe+Sn collisions at beam energies $E_{\text {beam }}=25-50 \mathrm{MeV}$ have been studied. Details on the shape of the distribution of the charge of the largest fragment, $Z_{\max }$, have been compared to a non-equilibrium aggregation model describing irreversible sol-gel transitions. The observed analogy between the two different phenomena has led the authors to conclude that multifragmentation is an aggregation-like phenomenon (similar to a condensation of vapor) occurring over shorter time-scales at higher energies due to the increasing importance of radial flow and with important links to the EoS.

\section{The equation of state of asymmetric nuclear matter in HIC}

In the last decade heavy-ion collision studies have explored the isospin degree of freedom in multifragmentation. The EoS for asymmetric nuclear matter (Asy-EoS), in the presence of asymmetry, $\mathrm{N} \neq \mathrm{Z}$, behaves differently than symmetric nuclear matter with $\mathrm{N}=\mathrm{Z}$. The nuclear Asy-EoS is parameterized as

$$
E(\rho, \delta)=E_{0}(\rho)+S(\rho) \cdot \delta^{2}
$$

where $E_{0}(\rho)$ is the EoS for symmetric nuclear matter. The function $S(\rho)$ is commonly referred to as symmetry energy term. The effects of isospin asymmetry $\mathrm{N} \neq \mathrm{Z}$, due to the symmetry energy, are proportional to the square of the isospin asymmetry parameter $\delta=\left(\rho_{n}-\rho_{p}\right) /\left(\rho_{n}+\rho_{p}\right)$. While the symmetric term $E_{0}(\rho)$ has been extensively studied [15], the density dependence of the symmetry energy, $S(\rho)$, is still largely unconstrained, especially at supra-saturation densities. Understanding this term also provides an improved understanding of masses [16], fission barriers, energies of isovector collective vibrations [17] and the thickness of the neutron skins of neutron-rich nuclei $[18,19]$. The symmetry energy plays a key role also in neutron star properties and type II supernovae [20]. Properties such as stellar radii and moments of inertia, crustal vibration frequencies [20], and neutron star cooling rates [20,21] depend significantly on $S(\rho)$. These phenomena are also investigated with ground and satellite observatories and X-ray studies [21]. One may expand the symmetry energy, $S(\rho)$, about the saturation density as $S(\rho)=S_{0}+\frac{L}{3}\left(\frac{\rho-\rho_{0}}{\rho_{0}}\right)+\frac{K_{s y m}}{18}\left(\frac{\rho-\rho_{0}}{\rho_{0}}\right)+\ldots$, where $L$ and $K_{\text {sym }}$ are the slope and the curvature parameters at $\rho_{0}$. The slope parameter, $L$, is related to $p_{0}$, the pressure due the symmetry energy for pure neutron matter at saturation density via $L=\left.3 \rho_{0}\left|d S_{\rho} / d \rho\right|\right|_{\rho_{0}}=\left[3 / \rho_{0}\right] / p_{0}[21]$. This symmetry pressure, $p_{0}$, is also related to the neutron skin thickness, $\delta R_{n p}$, of neutron rich heavy nuclei including ${ }^{208} \mathrm{~Pb}[18,19,21]$.

\section{Probes of the symmetry energy from heavy-ion collision dynamics}

In nuclear collisions it is useful to study asymmetric projectile and target nuclei (large N/Z asymmetries) in order to enhance the effects induced by $S(\rho)$ on nuclear dynamics and fragmentation, due to the $\delta^{2}$ factor on Eq. 1. Measured observables need to be compared to microscopic models where different Asy-EoS can be provided as inputs [22]. Several observables have been used more or less successfully to probe the symmetry energy at intermediate energies $(E / A=20-100 \mathrm{MeV})$ [23] . In this section I will describe only some of the most important ones: yield ratios of pre-equilibrium neutrons and protons in central collisions [23, 24]; isospin diffusion and drift phenomena in peripheral and mid-central collisions $[25,26]$.

\subsection{Probes from neutron-proton ratios in central collisions}

The relative yields of protons and neutrons are strongly related to the symmetry energy due to the fact that the symmetric part of the potential energy is repulsive for neutrons and attractive for protons [23]. 

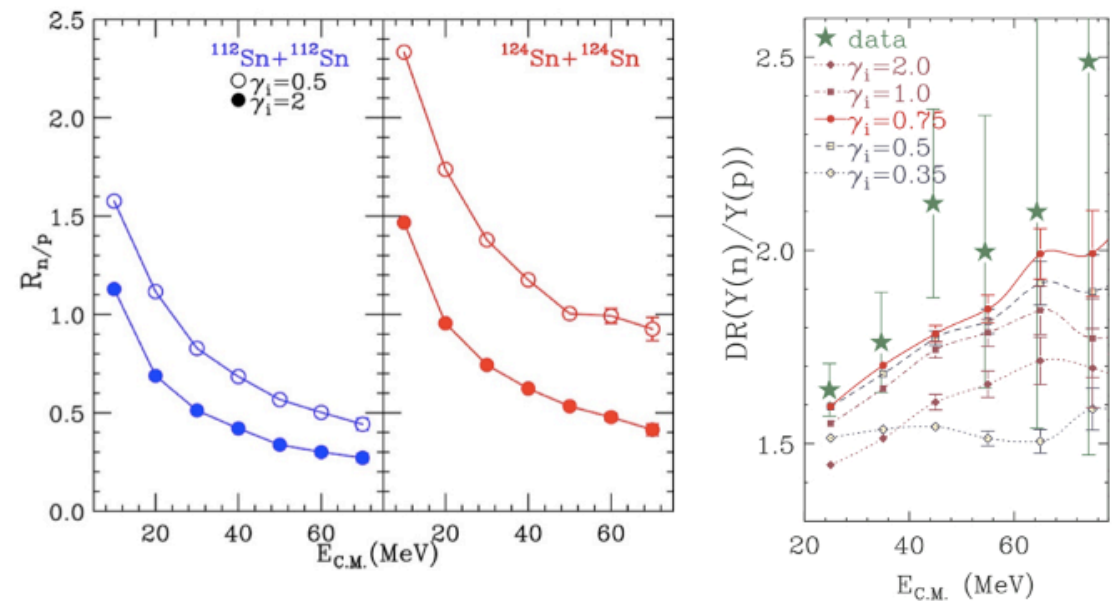

Figure 2. Left panels: neutron/proton yield ratios Vs. kinetic energy predicted by the ImQMD model [24] in ${ }^{112} \mathrm{Sn}+{ }^{112} \mathrm{Sn}$ (left, blue color) and ${ }^{124} \mathrm{Sn}+{ }^{124} \mathrm{Sn}$ (right, red color) and for different symmetry energies. Right panel: comparisons of double n-p ratios to ImQMD simulations [27].

This leads to different shapes of neutron and proton energy spectra depending on the stiffness of $S(\rho)$. The left side of Fig. 2 shows simulations performed with the ImQMD transport model (Ref. [24]). The ratio between protons and neutrons emitted around $90^{\circ}$ in the center of mass of ${ }^{112} \mathrm{Sn}+{ }^{112} \mathrm{Sn}$ (left panel, blue color) and ${ }^{124} \mathrm{Sn}+{ }^{124} \mathrm{Sn}$ (right panel, red color) as a function of their kinetic energies are shown. The parameterization of the symmetry energy used in these calculations is of the form: $S(\rho)=\frac{C_{c, k}}{2}\left(\frac{\rho}{\rho_{0}}\right)^{2 / 3}+\frac{C_{c, p}}{2}\left(\frac{\rho}{\rho_{0}}\right)^{\gamma_{i}}$ with the kinetic and potential parameters $C_{s, k}=25 \mathrm{MeV}, C_{s, p}=35.2 \mathrm{MeV}$ and the symmetry energy at saturation density, $S_{0}=S\left(\rho_{0}\right)=30.1 \mathrm{MeV}$. It is observed that the magnitude of the $R(n / p)$ ratio strongly depends on whether the density dependence of the symmetry energy is stiff $\left(\gamma_{i}=2.0\right)$ or soft $\left(\gamma_{i}=0.5\right)$. In order to remove residual, non-symmetry energy effects (due to Coulomb repulsion for protons and to secondary decays) experiments measure double $\mathrm{n} / \mathrm{p}$ ratios, $D R(n / p)$, de-

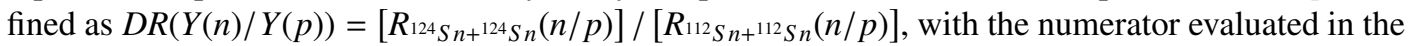
neutron-rich reaction system and the denominator evaluated in the neutron-poor reaction system [27]. Experimentally measured double ratios are displayed as star data points on the right side of Fig. 2 . The lines show double ratios obtained in model calculations with for $\gamma_{i}=0.35,0.5,0.75,1$ and 2 . These comparisons rule out both very soft and very stiff density-dependent symmetry terms, in favor of $\gamma_{i}$ values of about 0.7 .

\subsection{Probes from isospin diffusion and drift}

Peripheral collisions at Fermi energies mostly evolve towards a binary mechanism with the formation of quasi-projectile (QT) and quasi-target (QT) partners that exchange particles through a low density neck region. This exchange of particles is governed by isospin drift and isospin diffusion. Isospin drift consists of a net migration of neutrons from the high density QT and QP regions towards the low density neck region. It is thus due to the existence of density gradients. Isospin diffusion occurs when the two reacting nuclei have different N/Z asymmetries. It consists of an exchange of nucleons so as to equilibrate the overall $(\mathrm{N} / \mathrm{Z})$ of the total system. If the reaction time is long enough, the two reaction partners have enough time to achieve complete $\mathrm{N} / \mathrm{Z}$ equilibration by nucleon exchange. Otherwise 
the system re-separates early and the N/Z of the QP and QT will still reflect the original asymmetries of projectile and target at the entrance channel. These phenomena depend on reaction.time scales and reveal interesting chronology ordering in isotopically resolved fragment emission [28]. Isospin diffusion and drift are expected to be sensitive, respectively, to the strength of the symmetry energy and its derivative around saturation density $[25,26]$. They can have therefore been used as probes $S(\rho)[27-31]$.
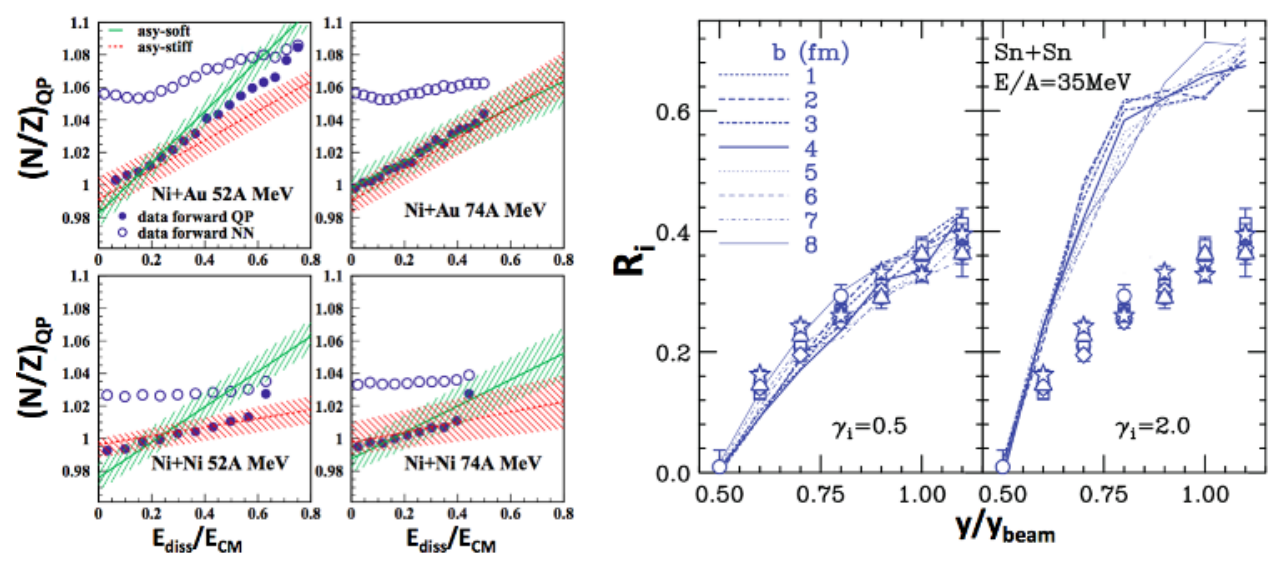

Figure 3. Isospin diffusion measurements and comparisons to model predictions in ${ }^{58} \mathrm{Ni}+{ }^{58} \mathrm{Ni}$ and ${ }^{58} \mathrm{Ni}+{ }^{197} \mathrm{Au}$ reactions at $\mathrm{E} / \mathrm{A}=52$ and $74 \mathrm{MeV}[30,31]$ (left side) and in ${ }^{112,124} \mathrm{Sn}+{ }^{112,124} \mathrm{Sn}$ collisions at $35 \mathrm{MeV} /$ nucleon (left side) and at $35 \mathrm{MeV} /$ nucleon [32] (right side). See text for descriptions.

In order to study isospin diffusion, experiments need to measure observables that are sensitive to the N/Z of the residual QP and QT systems. In order to isolate isospin diffusion effects from other effects not linked to the symmetry energy, one needs to study "mixed collisions", involving a neutronrich nucleus, A, and a neutron-deficient nucleus, B, and compare them to "symmetric collisions", involving $\mathrm{A}+\mathrm{A}$ and/or $\mathrm{B}+\mathrm{B}$. For example the Indra collaboration has reconstructed the $\mathrm{N} / \mathrm{Z}$ of the excited QP in ${ }^{58} \mathrm{Ni}+{ }^{58} \mathrm{Ni}$ and ${ }^{58} \mathrm{Ni}+{ }^{197} \mathrm{Au}$ reactions [30, 31]. In other experiments mirror nuclei yield ratios, such as $X_{i}=Y\left({ }^{7} \mathrm{Li}\right) / Y\left({ }^{7}\right) \mathrm{Be}$, were used as probes of the $\mathrm{N} / \mathrm{Z}$ asymmetry of their emitting sources [29, 32]. In this case the degree of isospin equilibration has been quantified by constructing an "imbalance ratio", as $R_{i}=\left[2 X_{i}-X_{A+A}-X_{B+B}\right]\left[X_{A+A}-X_{B+B}\right]$ [33]. $R_{i}=0$ if complete equilibration is achieved and $R_{i}<0$ in the case of incomplete equilibrium (N/Z or isospin translucency).

The data points on the left panel of Fig. 3 refer to the work on the INDRA collaboration that has compared a N/Z symmetric reaction, ${ }^{58} \mathrm{Ni}+{ }^{58} \mathrm{Ni}$, with an asymmetric reaction system, ${ }^{58} \mathrm{Ni}+{ }^{197} \mathrm{Au}$, at $\mathrm{E} / \mathrm{A}=52$ and $74 \mathrm{MeV}[30,31]$. The $\mathrm{N} / \mathrm{Z}$ of the quasi-projectile, $(\mathrm{N} / \mathrm{Z})_{Q} P$, has been reconstructed by using two different techniques (see Ref. [30] for details). The solid circles, obtained by collecting all forward emitted charged particle in the QP frame, represent the correlation between $(\mathrm{N} / \mathrm{Z})_{Q} P$ and the dissipated energy, $\mathrm{E}_{\text {diss }}$, normalized to the energy available in the center of mass of the reaction system, $\mathrm{E}_{C M}$. The ratio $\mathrm{E}_{\text {diss }} / \mathrm{E}_{C M}$ is directly linked to the impact parameter of the collision and to the interaction time between projectile and target. Larger values of $\mathrm{E}_{d i s s} / \mathrm{E}_{C M}$ arise from longer and more dissipative interactions with a more significant diffusion of neutrons and protons between the reaction partners. In the ${ }^{58} \mathrm{Ni}+{ }^{197} \mathrm{Au}$ reaction system, due to the large $\mathrm{N} / \mathrm{Z}$ difference between projectile and target nuclei, there is a net transfer of neutrons from the QT towards the QP. Then the N/Z ratio of the QP increases with increasing dissipation. The interaction time is longer at lower energies. 
Indeed a status of $\mathrm{N} / \mathrm{Z}$ equilibration seems to be achieved at $\mathrm{E} / \mathrm{A}=52 \mathrm{MeV}$, while $\mathrm{N} / \mathrm{Z}$ translucency is observed at higher energies and for less dissipative interactions. The extent to which N/Z equilibration is achieved is sensitive to the density dependence of the symmetry energy, as suggested by the hatched zones corresponding to simulations performed with the SMF (Stochastic Mean Field) code of Ref. [34] and the SIMON secondary decay code [35]. The simulated quantities are very close to the experimental data for all reaction systems, particularly for the asy-stiff case corresponding to a $\gamma_{i}$ exponent close to unity $\left(\gamma_{i} \approx 1\right)$.

The right panel of Fig. 3 refers to a work performed with the Chimera $4 \pi$ array to study ${ }^{112,124} \mathrm{Sn}+{ }^{112,124} \mathrm{Sn}$ collisions at $\mathrm{E} / \mathrm{A}=35 \mathrm{MeV}$ [32]. In this case the $\mathrm{N} / \mathrm{Z}$ of the decaying system is probed by using the imbalance ratios, $R_{i}$, constructed with the mirror nuclei yield ratios $X_{i}=\frac{\left.Y{ }^{7} L i\right)}{\left.Y Y^{7} B e\right)}$. The different symbols refer to collisions at different impact parameters, selected by using the multiplicity of charged particles, and show $R_{i}$ as a function of ${ }^{7} \mathrm{Li}$ and ${ }^{7} \mathrm{Be}$ rapidities, $y$, normalized to the rapidity of the beam, $y_{\text {beam }}$. The results seem to show that a complete $\mathrm{N} / \mathrm{Z}$ equilibration is not achieved even in central collisions. This result seems to be not in agreement with the finding of the INDRA collaboration on $\mathrm{Ni}+\mathrm{Au}$ collisions discussed on the left panel of the same figure and showing an achievement of $\mathrm{N} / \mathrm{Z}$ equilibration for the most dissipative collisions at $\mathrm{E} / \mathrm{A}=52 \mathrm{MeV}$. This discrepancy may result from the fact that central collisions selections used in Ref.[32], based only on the multiplicity of charged particles, are less accurate than those used in Indra data Ref. [30]. On the other hand, the observation of isospin translucency around projectile rapidity $\left(y / y_{\text {beam }} \approx 1\right)$ in midperipheral and peripheral collisions is robust and can be used as well to probe the density dependence of the symmetry energy. This is accomplished in Ref. [32] by means of comparisons to ImQMD (Improved Quantum Molecular Dynamics) model calculations. Simulations with different stiffness of the symmetry energy seem to exclude high values for the stiffness parameter $\gamma_{i} \approx 2$, providing better agreement with the data for $\gamma_{i}<1$ [32], consistently with the results of [30,31]. These studies suggest that it is important attempt to describe observables measured in different experiments with the same reaction model. Similarly it is interesting to understand whether a unique parameterization of $S(\rho)$ can describe observables measured in the same experiment by using different reaction models. Such an attempt has been carried out in Ref. [36] where the measured effects of the symmetry energy on directed flow at Fermi energies where compared to three different models based on different recipes to describe the dynamics of HIC. These attempts are very important in order to constrain the density dependence of the symmetry energy reducing model dependencies.

\section{Symmetry energy: present status and future perspectives}

Fig. 4 shows the present status of measurements on the density dependence of the symmetry energy. These figures represent the result of efforts by a community [37]. The left panel shows different parameterizations of $S(\rho)$ proposed in the literature (solid lines). The soft blue band correspond to the range of densities and $S(\rho)$ values that are presently constrained by heavy-ion collision measurements. In a different representation using the strength, $S_{0}$, and the slope, $L$, of $S(\rho)$ at $\rho=\rho_{0}$ (see right panel on Fig.4), one can compare constrains obtained from different experimental techniques (including those used in astrophysics). The wide shaded light-blue area represents the constraints on $S_{0}$ and $L$ obtained from isospin diffusion and neutron-proton double yield ratios measured in $\mathrm{Sn}+\mathrm{Sn}$ collisions at $50 \mathrm{MeV} /$ nucleon [27] (Section 4). The red box and star data symbols report the results obtained, respectively, from transverse flow [36] and isospin diffusion measurements [32] at $35 \mathrm{MeV} /$ nucleon. As indicated in the figure other studies have extracted $L$ and $S_{0}$ from ground-state properties, such as nuclear masses (FRDM, box with pink solid line) and ${ }^{2} 08 \mathrm{~Pb}$ neutron skin thickness ( $\operatorname{see} \operatorname{Pb}(\vec{p}, \vec{p})$, right box with blue dashed line), and from excited state properties, such as the energies of isobaric analog 

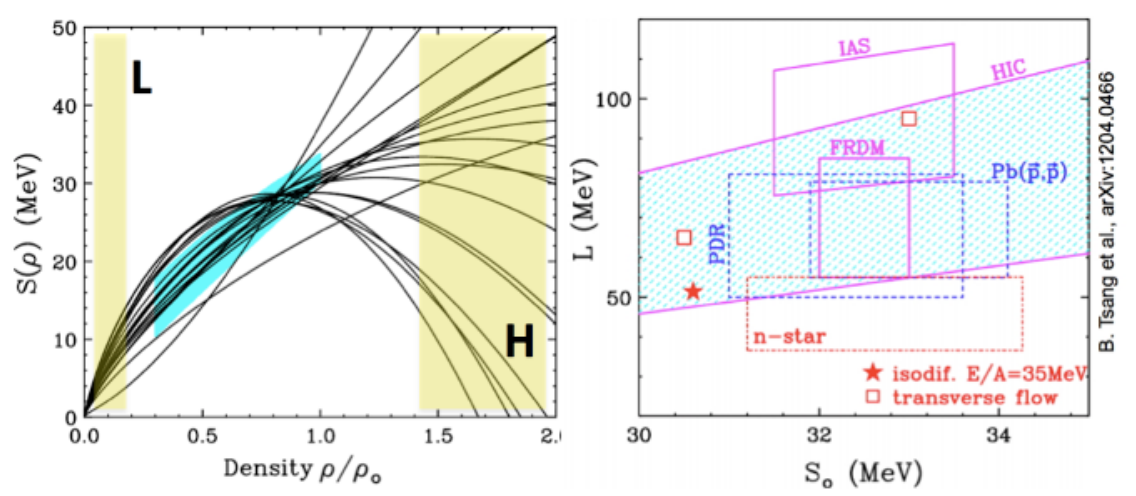

Figure 4. Left pane: Different theoretical prediction of $S(\rho)$ (lines) and regions constrained by existing HIC data (shaded-color area, from Ref. [37]). Right panel: status of measurements of $L$ and $S_{0}$ parameters (see text for details) from different experimental approaches.

states (IAS) or from collective oscillation measurements, such as the energies and strengths of giant and pygmy dipole resonances (PDR). For a review of such techniques see Ref. [37]. The red dashed line box shows also the present constraints coming from observational astrophysics [38]. It will be an important scientific objective that both laboratory measurements and astrophysical observations can be described consistently with the same assumptions about the density dependence of the symmetry energy.

Concerning the status of heavy-ion collision measurements, some important goals should be considered for the future. It will be important to improve experimental investigations in order to reduce the error bars and the widths of the shaded areas on Fig. 4. This goal implies improved characterization of collision events with more efficient and performing $4 \pi$ detector arrays, and integration of neutron detection capabilities. The regions indicated with "L" on the left panel of Fig. 4 correspond to studies of the symmetry energy at very low densities where the presence of clustering phenomena may reveal new insights [39]. On the other "H" side of the plot (supra-saturation densities), experimental probes of $S(\rho)[23,40]$ include neutron/proton elliptic flow, neutron/proton energy spectra, meson production and ratios (such as $\pi^{+} / \pi^{-}$and $K^{+} / K^{0}$ ratios), with a special attention to the problem of effective proton/neutron mass splitting that might affect predictions of the symmetry energy, especially at high energies [23, 40]. Experiments at future facilities such as Fair (Germany), FRIB (USA), Riken (Japan) and RISP (South Korea), with new dedicated detector setups will provide tools to constrain the symmetry energy at higher densities. Efforts to improve comparisons of theoretical predictions to experimental data are required. In particular the possibility of performing extensive simulations where collision events are selected (based on impact parameter) and analyzed in a consistent way as in experimental data would be highly beneficial to making further progress in constraining the density dependence of the symmetry energy.

\section{References}

[1] G. Sauer et al., Nucl. Phys. A264, 221 (1976)

[2] H.R. Jaquaman et al., Phys. Rev. C29, 2067 (1984)

[3] B. Borderie and M.-F. Rivet, Prog. Part. Nucl. Sci. 61, 551 (2008) 
[4] Y. Aoki, G. Endrodi, Z. Fodor, S. Katz, and K. Szabo, Nature 443, 675 (2006)

[5] C.L. Zhou et al., Eur. Lett. 98, 66003 (2012).

[6] M. Colonna et al., Nucl. Phys. A 580, 312 (1994); M. Colonna et al., Nucl. Phys. A 630, 136c (1998); P. Chomaz et al., Phys. Rep. 389 (2004) 263.

[7] World Consensus Initiative (WCI) book "Dynamics and Thermodynamics with Nucleonic Degrees of Freedom", Eur. Phys. J. A30 (2006).

[8] R.T. de Souza et al., Eur. Phys. J. A30 275 (2006)

[9] J. Pochodzalla et al., Phys.Rev.Lett. 75 (1995); J.A.Hauger et al., Phys.Rev.Lett. 77, 235 (1996); Y.G. Ma et al., Phys. Lett. B390, 41 (1997); B. Borderie et al., Phys. Lett. B723, 140 (2013).

[10] P.F. Mastinu et al., Phys. Rev. Lett. 76, 2646 (1996)

[11] M. Pichon et al., Nucl. Phys. A779, 267 (2006); M. Bruno et al., Nucl. Phys. A807, 48 (2008); E. Bonnet et al., hys. Rev. Lett. 103 (2009) 072701.

[12] J.B. Elliott et al., Phys. Rev. Lett., 88, 042701 (2002); J. D. Frankland et al., Phys. Rev. C71, 034607 (2005).

[13] J. Aichelin and J. Huefner, Phys. Lett. B136, 15 (1984).

[14] D. Gruyer et al., Phys. Rev. Lett. 110, 172701 (2013).

[15] P. Danielewicz, R. Lacey, W.G. Lynch, Science 298, (2002) 1592.

[16] P. Danielewicz, Nucl. Phys. A 727 (2003) 233 and references therein; P. Danielewicz and J. Lee, Nucl. Phys. A818, 36 (2009).

[17] A. Klimkiewicz et. al., Phys. Rev. C 76 (2007) 051603.

[18] B.A. Brown et al., Phys. Rev. Lett. 85 (2000) 5296.

[19] C. J. Horowitz and J. Piekarewicz, Phys. Rev. Lett. 86 (2001) 5647.

[20] J.M. Lattimer, M. Prakash, Science 304, (2004) 536.

[21] A.W. Steiner et al., Phys. Rep. 411 (2005) 325 and references therein.

[22] C. Fuchs et al., Eur.Phys.J. A30, 5 (2006); C. Fuchs, Prog. Part. Nucl. Phys. 56, 1 (2006).

[23] Bao-An Li et al., Phys. Rep.464, 113 (2008); V. Baran et al., Phys. Rep. 410 (2005) 355.

[24] Y. Zhang et al., Phys. Lett. B664, 145 (2008)

[25] M. Colonna et al., Eur. Phys. J. A30, 182 (2006)

[26] L. Shi et al., Phys. Rev. C68, 064604 (2003)

[27] M.B. Tsang et al., Phys. Rev. Lett. 102, 122701 (2009)

[28] E. De Filippo et al., Phys. Rev. C86, 014610 (2012).

[29] M.B. Tsang et al., Phys. Rev. Lett. 102, 122701 (2009)

[30] E. Galichet et al., Physical Review C79, 064614 (2009).

[31] E. Galichet et al., Physical Review C79, 064615 (2009).

[32] Z.Y. Sun et al., Phys. Rev. C82, 051603 (2010).

[33] F. Rami et al., Phys. Rev. Lett. 84, 1120 (2000); J. Rizzo et al., Nucl. Phys. A 806, 79 (2008).

[34] J. Rizzo, M. Colonna, and M. Di Toro, Phys. Rev. C 72, 064609 (2005).

[35] D. Durand, Nucl. Phys. A541, 266 (1992).

[36] Z. Kohley et al., Phys. Rev. C82, 064601 (2010); Z. Kohley et al., Phys. Rev. C85, 064605 (2012)

[37] M.B Tsang et al., arXiv:1204.0466 [nucl-ex]

[38] A.W. Steiner et al., ApJ. 722, 33, (2010)

[39] R. Wada et al., PRC85, 064618 (2012)

[40] M. Di Toro et al., Prog. in Part. and Nucl. Phys., 62, 389 (2008) 